\title{
Aspectos da alimentação de Knodus heteresthes (Characiformes, Characidae) no igarapé do Nove, bacia do rio Machado, Rondônia, Brasil
}

\author{
Wesclen Vilar Nogueira * \\ Igor David da Costa \\ Laboratório de Ciências Ambientais, Departamento de Engenharia de Pesca \\ Universidade Federal de Rondônia, Campus Presidente Médici \\ Rua da Paz, 4376, Bairro Lino Alves Teixeira, CEP 76.916-000, Presidente Médici - RO, Brasil \\ * Autor para correspondência \\ wesclenvilar@gmail.com
}

Submetido em 27/09/2013

Aceito para publicação em 07/04/2014

\section{Resumo}

Neste estudo, descrevemos o hábito alimentar de Knodus heteresthes em um igarapé amazônico e exploramos a hipótese de que as variações da dieta ocorrem em função da alternância de estações e desenvolvimento ontogenético. A maioria dos espécimes coletados apresentou tamanho pequeno $(<30 \mathrm{~mm})$. Observamos que a espécie consome ampla variedade de itens, variando de material de origem vegetal, como algas, sementes e folhas, e material de origem animal, como aracnídeos, briozoários e insetos terrestres e aquáticos em diversos estágios de vida, sugerindo uma dieta onívora, embora com expressivo consumo de insetos alóctones. Itens de origem alóctone foram mais representativos do que itens autóctones para jovens $\left(\chi^{2}=70,4 ; p<0,05\right)$ e adultos $\left(\chi^{2}=60,6 ; p<0,05\right)$, em ambas as estações (chuvosa, $\chi^{2}=53,0 ; p<0,05$; seca, $\chi^{2}=77,0 ; p<0,05$ ), e representaram mais de $70 \%$ da dieta da espécie em todas as amostras, indicando certo grau de especialização. Tal especialização, por sua vez, pode estar relacionada às elevadas abundâncias encontradas pela espécie, que possivelmente demonstra boa capacidade em alocar energia para crescimento, no caso dos indivíduos jovens, e reprodução, no caso dos adultos, tornando-a uma das espécies dominantes em igarapés conservados.

Palavras-chave: Amazônia; Dieta; Ontogenia; Sazonalidade

\section{Abstract}

Feeding aspects of Knodus heteresthes (Characiformes, Characidae) in igarapé of the Nove Machado river basin, Rondônia, Brazil. In this study, we describe the feeding habit of Knodus heteresthes in an Amazonian igarapé and explore the hypothesis that variations in diet are due to alternation of seasons and ontogenetic development. Most specimens collected were small-sized $(<30 \mathrm{~mm})$. We observed that the species eats a wide variety of items, ranging from vegetal material, such as algae, seeds, and leaves, and animal material, such as arachnids, bryozoans, and terrestrial and aquatic insects at various life stages, suggesting an omnivorous diet with a significant consumption of allochthon insects. Items of allochthon origin were more representative than autochthon items for young $\left(\chi^{2}=70.4 ; p<0.05\right)$ and adults individuals $\left(\chi^{2}=60.6 ; p<0.05\right)$, in both seasons (rainy, $\chi^{2}=53.0 ; p<0.05$; dry, $\chi^{2}=77.0 ; p<0.05$ ), and they accounted for over $70 \%$ of the species' diet in all samples, indicating some degree of specialization. Such specialization, in turn, can be related to the high 
abundances found by the species, which possibly show a good ability to allocate energy to growth, in the case of young individuals, and reproduction, in the case of adults, making it a dominant species in preserved igarapés.

Key words: Amazon; Diet; Ontogeny; Seasonality

\section{Introdução}

Os peixes de água doce neotropicais representam um oitavo da diversidade de vertebrados viventes e constituem a maior de todas as faunas epicontinentais do planeta (VARI; MALABARBA, 1998). O Brasil tem a maior diversidade de espécies de peixes de água doce do mundo, com elevada riqueza e endemismo em muitos grupos. Esta grande biodiversidade esta relacionada aos grandes sistemas de rios, composto por bacias e regiões com muitos ecossistemas diferenciados (BUCKUP et al., 2007; ABELL et al., 2008; LÉVÊQUE et al., 2008). A elevada representatividade da família Characidae, tanto em número de espécies quanto em número de indivíduos, é consistente com o padrão geral encontrado por outros autores para a região Neotropical (CASTRO; MENEZES, 1998; BRITSKI et al., 1999; LOWE-MCCONNELL, 1999; SHIBATTA et al., 2002; REIS et al., 2003; BUCKUP et al., 2007). Nesta família é encontrado o principal conjunto de espécies nectônicas residentes em riachos de médio e baixo gradiente (BUCKUP, 1999), predominando inclusive em termos de abundância e biomassa (CASTRO et al., 2003; 2004; 2005) e desempenhando um papel importante na produção de peixes de riachos (CENEVIVA-BASTOS; CASATTI, 2007).

Apesar dessa importância, pouco se conhece a respeito da ecologia das espécies de Characidae, especialmente sobre como alterações de origem antrópica nos ecossistemas podem influenciar a utilização de recursos alimentares e a sobrevivência dessas espécies (CENEVIVA-BASTOS; CASATTI, 2007). Na bacia do rio Machado, um importante afluente do rio Madeira situado no estado de Rondônia, amostragens recentemente realizadas em 75 igarapés registraram a espécie Knodus heteresthes entre as 10 espécies dominantes (CASATTI et al., 2013). Apesar do destaque que a espécie ocupa em termos de abundância, estudos sobre sua biologia ainda não foram realizados, assim como para muitas outras espécies de peixes desta região. Neste trabalho, objetivamos estudar a biologia alimentar de Knodus heteresthes, a fim de responder (1) quais os itens alimentares preferenciais, (2) se há influência da sazonalidade na dieta da espécie e (3) e se há mudança na dieta a partir de variações ontogenéticas.

\section{Material e Métodos}

\section{Área de estudo}

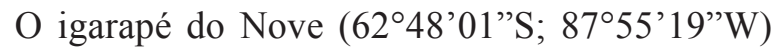
está localizado na bacia de drenagem do rio Machado, situada na porção leste do estado de Rondônia (Figura 1). O clima da região é caracterizado por temperaturas que variam entre 19 e $33^{\circ} \mathrm{C}$ e precipitação anual em torno de $2500 \mathrm{~mm}$ (KRUSCHE et al., 2005), sendo o regime pluviométrico caracterizado por uma estação seca (final de maio a setembro) e uma chuvosa (outubro a abril) (FERNANDES; GUIMARÃES, 2002). O igarapé estudado apresenta profundidade média de $0,26 \pm 0,05$ $\mathrm{m}$, largura média de $6,92 \pm 2,40 \mathrm{~m}$, velocidade média de $0,20 \pm 0,10 \mathrm{~m} / \mathrm{s}$, substrato arenoso, presença de gramíneas e plantas aquáticas de pequeno porte. A microbacia do igarapé estudada apresenta pasto na matriz adjacente, contudo é predominante a presença de angiospermas arbóreas localizadas às margens do igarapé na região, à montante e à jusante do trecho amostrado. No trecho onde foram realizadas as coletas, foi observado um menor número de árvores (aproximadamente dois indivíduos $/ \mathrm{m}^{2}$ ) quando comparado às áreas à montante e à jusante. 
FIGURA 1: Mapa da bacia do rio Machado com a localização da área de estudo (circulo preto).

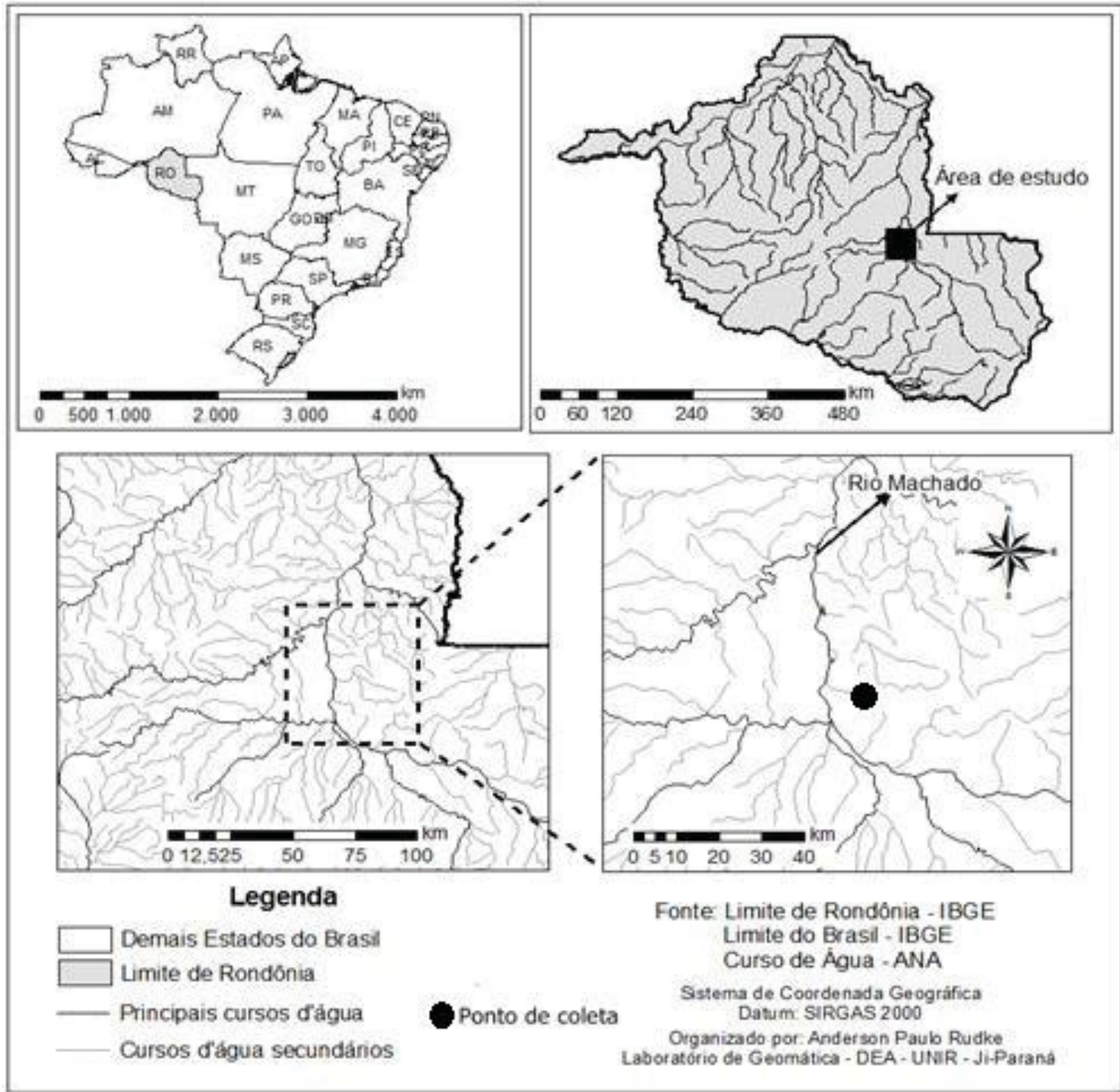

\section{Amostragem e análise de dados}

As coletas foram realizadas bimestralmente de outubro 2011 a setembro 2012. Em um trecho de 50 $\mathrm{m}$ foram utilizados rede de arrasto de mão (picaré) de 2,0 x 1,20 m com malha de $2 \mathrm{~mm}$ e um puçá com abertura de $0,46 \times 0,33 \mathrm{~m}$ com malha de $2 \mathrm{~mm}$ durante uma hora. Como complemento, utilizamos 10 armadilhas tipo covo, confeccionadas com garrafa pet $(2 \mathrm{~L}$ de volume), contendo milharina (milho moído) como isca, que permaneceram armadas durante $24 \mathrm{~h}$ com revisões a cada $4 \mathrm{~h}$. As armadilhas permaneceram equidistantes $2 \mathrm{~m}$ entre si, ao longo de todo o trecho, com a abertura voltada para jusante. Antes das coletas, as extremidades dos trechos de amostragem foram bloqueadas com redes de malha fina (5 $\mathrm{mm}$ entre nós opostos) para evitar a fuga dos peixes. Os espécimes coletados foram fixados em formol $10 \%$ e preservados em etanol a $70 \%$.

Foram realizadas sessões de mergulho a fim de promover observações comportamentais da espécie $K$. heteresthes. As observações foram realizadas utilizando os métodos ad libitum, animal focal (LEHNER, 1999) e gravações com uma máquina digital, modelo Nikon Coolpix AW100 camuflada, totalizando 10 horas de observação. As observações ocorreram no trecho central do igarapé (maior velocidade da correnteza) durante quatro horas no período diurno (07h00min-11h00min). Para cada indivíduo ou grupo de indivíduos observados em atividade foram registrados: 
posição no canal (margem ou canal principal e posição na coluna de água) e características comportamentais. As informações sobre a espécie foram registradas com lápis em placas de polietileno de $15 \times 20 \mathrm{~cm}$ durante as sessões de mergulho.

O comprimento padrão $(\mathrm{CP})$ de cada exemplar foi medido para definição das classes de tamanho (CT). Os conteúdos estomacais foram acondicionados em álcool $70^{\circ} \mathrm{GL}$ e os itens alimentares identificados com o auxílio de microscópio estereoscópico, até o menor nível taxonômico possível. Para a análise da alimentação foram utilizados os métodos de frequência de ocorrência ( $\mathrm{F} i \%)$ e volumétrico (Vi\%) (HYNES, 1950; HYSLOP, 1980). O volume de cada item foi obtido através de uma placa de petri milimetrada, onde foi determinada a área ocupada pelo item em milímetros cúbicos sendo posteriormente convertido em mililitros (HELLAWELL; ABEL, 1971). Esses dados foram combinados no Índice de importância alimentar (IA $i$ ) proposto por Kawakami e Vazzoler (1980) e adaptado por Hahn et al. (1997), de acordo com a equação: $\mathrm{IA} i=(\mathrm{Fi} * \mathrm{Vi}) / \Sigma\left(\mathrm{Fi}{ }^{*} \mathrm{Vi}\right) * 100$, onde $\mathrm{i}=1,2, \ldots \mathrm{n}$ itens alimentares; $\mathrm{Fi}=$ frequência de ocorrência de um determinado item alimentar; $\mathrm{Vi}=$ volume de um determinado item alimentar. Os itens alimentares foram analisados de acordo com a origem (autóctones e alóctones), estação do ano (estação chuvosa e seca) e fases de vida (jovens e adultos).

O estabelecimento das classes de tamanho (CT) que reuniu os exemplares em jovens e adultos foi realizado através da observação do estádio de maturação gonadal (baseado em NUÑEZ; DUPONCHELLE, 2008) de 150 exemplares, sendo que os indivíduos com CP superior a $30 \mathrm{~mm}$ apresentaram gônadas maduras (adultos). Observamos a presença de ovários grandes e cheios ocupando significativo espaço na cavidade abdominal, com elevada presença de ovócitos amarelados e com formato ovulado. Para os machos, foram observados testículos mais largos, cheios e completamente brancos. Para a realização das análises estatísticas os dados foram previamente log-transformados $(\log \mathrm{x}+1)$. Pelo fato dos pressupotos de normalidade $\mathrm{e}$ homocedasticidade não serem alcançados, foi utilizado um teste não-paramétrico (Kruskal-Wallis) afim de testar as diferenças entre as CT. O teste de $\chi^{2}$ foi aplicado a fim de detectar diferenças na participação dos itens alimentares de origem autóctone e alóctone entre jovens e adultos, e entre as estações (seca vs. chuvosa). Todas as análises foram realizadas no programa Statistica 7.0. Os resultados dos testes foram considerados significativos sempre que $p \leq 0,05$.

\section{Resultados}

Um total de 90 exemplares foi analisado, para a estação chuvosa a CT mais representativa em abundância foi de 26 a $30 \mathrm{~mm}(\mathrm{n}=25)$, seguido de 21 a $25 \mathrm{~mm}$ $(\mathrm{n}=9)$ e 31 a $35 \mathrm{~mm}(\mathrm{n}=7)$. As CT inferiores e superiores aos resultados supracitados apresentaram abundância inferior a três indivíduos. Na estação seca a maior abundância $(\mathrm{n}=19)$ foi da CT de 21 a $25 \mathrm{~mm}$, seguido de exemplares com 15 a $20 \mathrm{~mm}(\mathrm{n}=12)$ e de 26 a $30 \mathrm{~mm}(\mathrm{n}=9)$. As classes de tamanho superiores apresentaram abundância menor que cinco indivíduos (Figura 2).

Somente as CT de 15 a $20 \mathrm{~mm}$ e 26 a $30 \mathrm{~mm}$ apresentaram diferença significativa entre as estações seca e chuvosa. Os indivíduos compreendidos na CT de 15 a $20 \mathrm{~mm}$ foram mais abundantes na estação seca $(\mathrm{n}=12)$ do que na chuvosa $(\mathrm{n}=1)(\chi 2=10,1 ; \mathrm{p}<0,05)$ e para a CT de 26 a $30 \mathrm{~mm}$ um maior número de indivíduos foi coletado na estação chuvosa $(n=25)$ do que na seca $(n=9)\left(\chi^{2}=7,52 ; p<0,05\right)$. Diferenças significativas no número de indivíduos entre as $\mathrm{CT}$ não foram encontradas (Kruskall-Wallis, $\chi^{2}=0,00 ; p>0,05$ ).

A espécie $K$. heterestes ingeriu muitos itens, variando de material de origem vegetal, como sementes, e material de origem animal, como aracnídeo, bryozoários e insetos terrestres e aquáticos em diversos estágios de vida. O item fragmento de insetos terrestres foi o mais consumido por jovens (chuvosa, IA $i \%=77,2$; seca, IA $i \%=89,9$ ) e adultos (chuvosa, IA $i \%=72,2$; seca, IA $i \%=93,5)$ em ambas as estações. Dentre os demais itens, pupas de Diptera (IA $i \%=15,3$ ) e Hymenoptera (IA $i \%=3,9$ ), na estação chuvosa, e Coleoptera (IA $i \%=1,7)$, na estação seca, foram os mais representativos na dieta de indivíduos adultos. 
FIGURA 2: Frequência absoluta (n) dos indivíduos de Knodus heteresthes coletados no igarapé do Nove por classe de tamanho e estação.

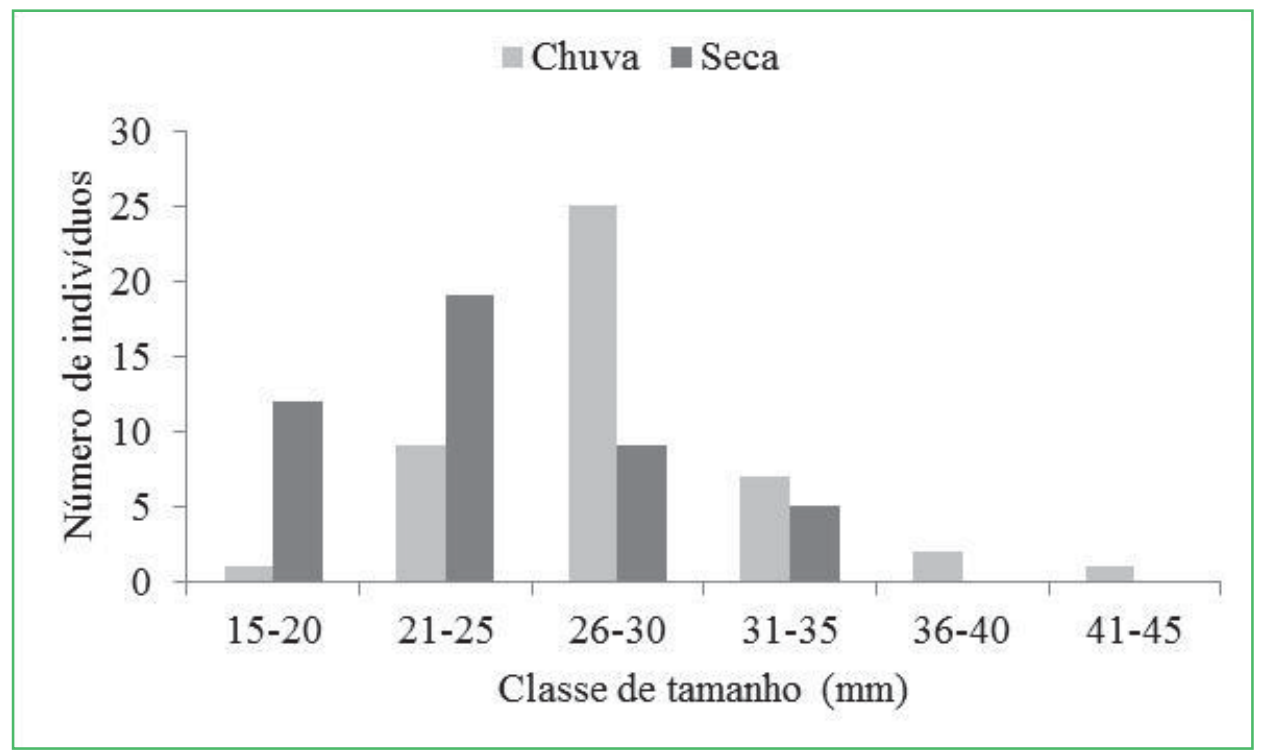

Hymenoptera (IA $i \%=9,3)$ e larvas de Chironomidae grupo de itens mais abundantes na alimentação de jovens (chuvosa, IA $i \%=2,43$; seca IA $i \%=4,2$ ) foram o segundo para os períodos de chuva e seca, respectivamente (Tabela 1).

TABELA 1: Percentual do índice alimentar (IAi\%) de cada item consumido por indivíduos jovens e adultos de $K$. heterethes coletados nas estações seca e chuvosa no igarapé do Nove, Bacia do rio Machado, Rondônia. * = itens alóctones, $* *=$ itens autóctones $\mathrm{e}^{* * *}=$ itens de origem indeterminada.

\begin{tabular}{|c|c|c|c|c|}
\hline \multirow{2}{*}{ Itens alimentares } & \multicolumn{2}{|c|}{ Jovens } & \multicolumn{2}{|c|}{ Adultos } \\
\hline & Chuvosa & Seca & Chuvosa & Seca \\
\hline \multicolumn{5}{|l|}{ Origem Vegetal } \\
\hline Sementes* & 1,49 & 0,22 & 0,35 & 0,13 \\
\hline Material Vegetal não identificado*** & 0,34 & 0,02 & 0,00 & 0,00 \\
\hline \multicolumn{5}{|l|}{ Origem Animal } \\
\hline Aracnideo terrestre* & 0,09 & 0,00 & 0,00 & 0,00 \\
\hline Bryozoa** $^{* *}$ & 0,00 & 0,08 & 0,00 & 0,00 \\
\hline Coleoptera*** & 1,02 & 0,19 & 1,85 & 1,71 \\
\hline Diptera* & 0,00 & 0,07 & 1,38 & 0,00 \\
\hline Ephemeroptera** & 0,26 & 0,09 & 0,92 & 0,00 \\
\hline Hemiptera** & 0,13 & 0,12 & 0,00 & 0,00 \\
\hline Hirudinae $* *$ & 0,00 & 0,40 & 0,00 & 0,00 \\
\hline Homoptera* & 0,00 & 0,00 & 0,35 & 0,00 \\
\hline Hymenoptera* & 9,03 & 1,64 & 3,92 & 0,00 \\
\hline Isoptera* & 0,30 & 0,00 & 0,00 & 0,00 \\
\hline Orthoptera* & 0,00 & 0,00 & 0,00 & 0,76 \\
\hline Plecoptera $* *$ & 0,09 & 0,02 & 0,00 & 0,00 \\
\hline Pupa de Chironomidae** & 0,09 & 0,01 & 0,00 & 0,00 \\
\hline
\end{tabular}




\begin{tabular}{|c|c|c|c|c|}
\hline Pupa de Diptera** & 0,09 & 0,40 & 15,34 & 0,00 \\
\hline Larva de Ceratopogonideae** & 1,62 & 1,68 & 0,23 & 0,13 \\
\hline Larva de Chironomidae** & 2,43 & 4,22 & 0,46 & 0,38 \\
\hline Larva de Coleoptera** & 0,06 & 0,00 & 0,00 & 0,00 \\
\hline Larva de Diptera** & 0,02 & 0,52 & 0,35 & 0,38 \\
\hline Larva de Lepidoptera** & 0,00 & 0,03 & 0,35 & 0,82 \\
\hline Juvenil de Odonata** & 0,06 & 0,09 & 0,00 & 0,00 \\
\hline Larva de Simuliidae** & 0,00 & 0,01 & 0,58 & 0,00 \\
\hline Larva de Trichoptera** & 3.07 & 0,08 & 0,00 & 1,58 \\
\hline Fragmentos de insetos terrestres* & 77,27 & 89,95 & 72,20 & 93,50 \\
\hline Inseto não Identificado*** & 0,00 & 0,01 & 0,00 & 0,00 \\
\hline Ovos não Identificados*** & 1,56 & 0,16 & 1,96 & 0,63 \\
\hline
\end{tabular}

O consumo de itens autóctones diferiu significativamente do de itens alóctones tanto entre os indivíduos jovens $(\chi 2=70,4 ; p<0,05)$ como entre os adultos $(\chi 2=60,6 ; p<0,05)$, sendo os itens de origem alóctone os mais consumidos em ambas as fases de vida (Figura 3).
O consumo de itens autóctones diferiu significativamente do de itens alóctones tanto na estação chuvosa $\left(\chi^{2}=53,0 ; p<0,05\right)$ como na estação seca $\left(\chi^{2}=\right.$ $77,0 ; p<0,05)$, sendo os itens de origem alóctone os mais consumidos em ambas as estações (Figura 4).

FIGURA 3: Percentual do índice alimentar dos itens autóctones e alóctones consumidos por jovens e adultos de K. heteresthes no igarapé do Nove.

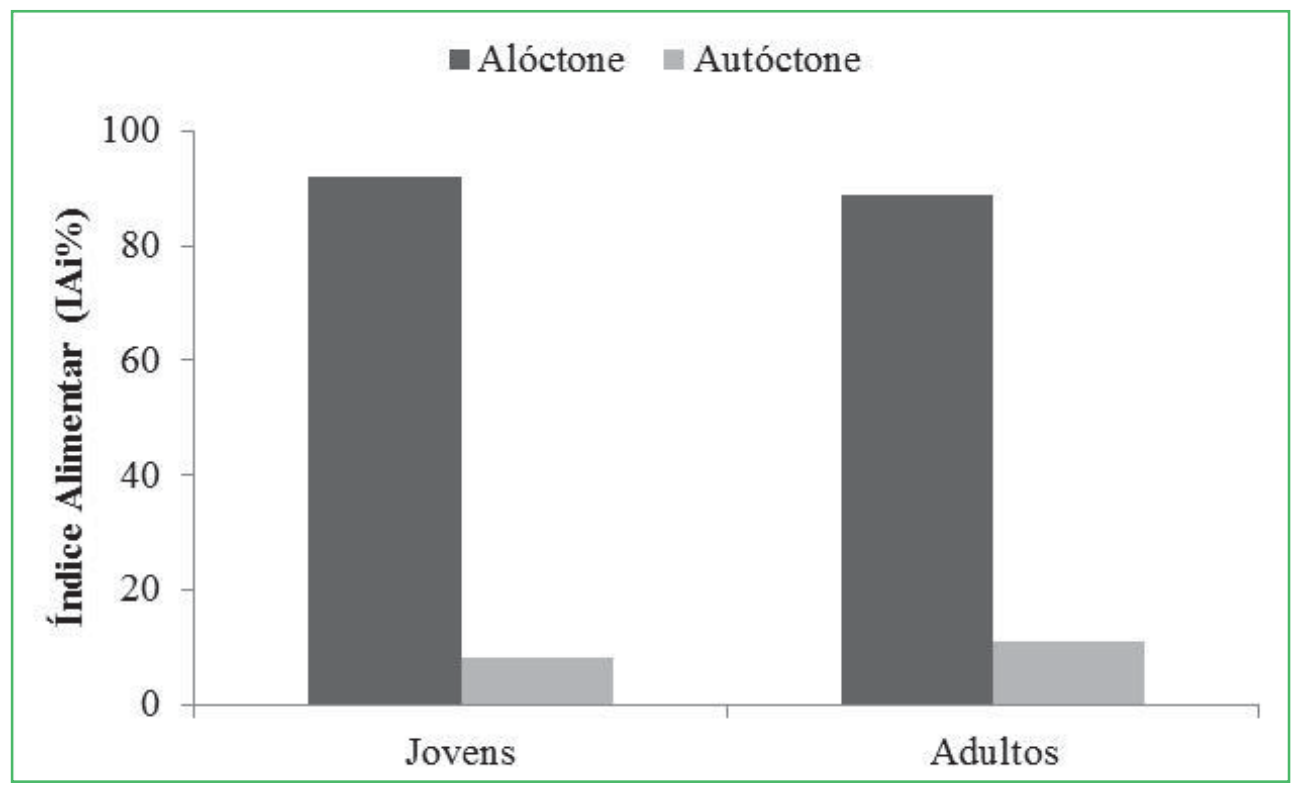


FIGURA 4: Percentual do índice alimentar dos itens autóctones e alóctones consumidos por $K$. heteresthes no igarapé do Nove durante as estações chuvosa e seca.

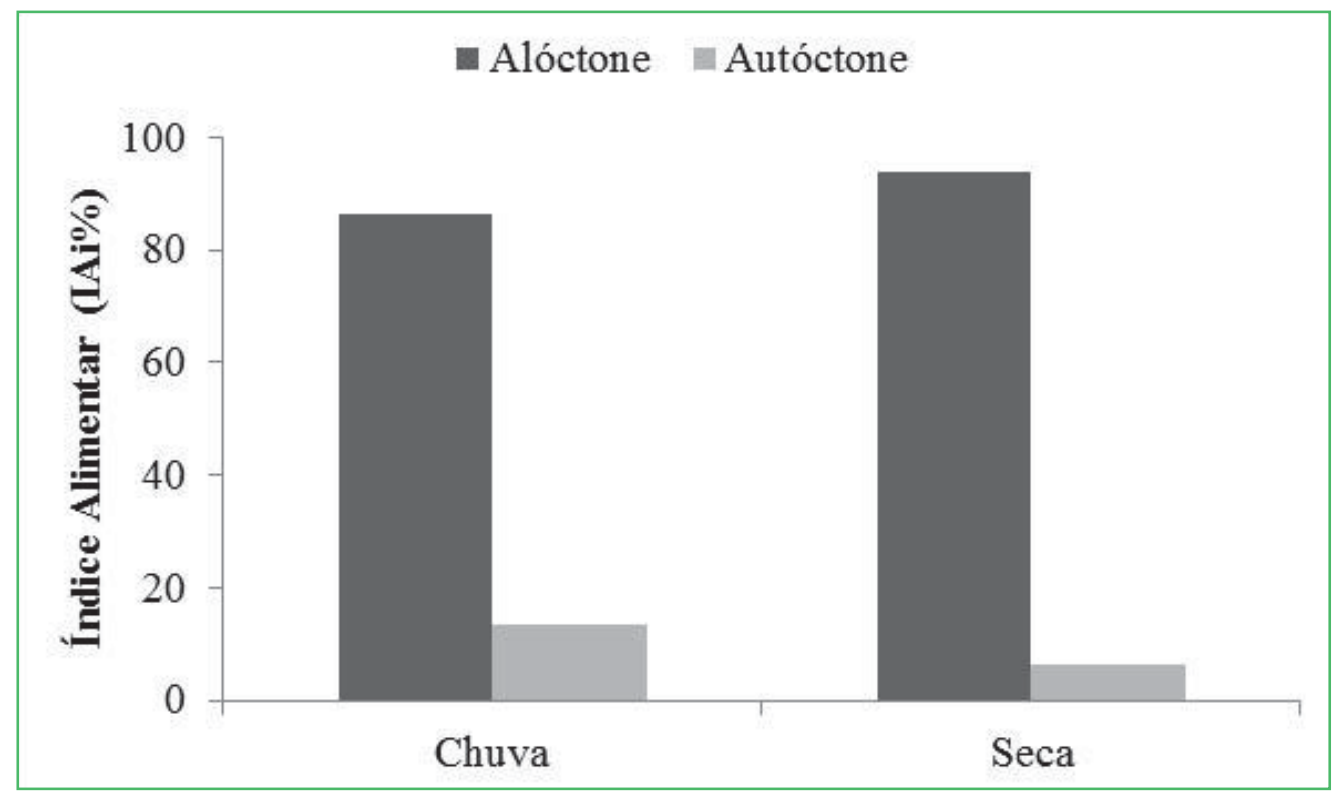

Grupos de 10-22 indivíduos de $K$. heteresthes de maior porte foram observados forrageando continuamente, praticando a captura de itens na coluna d'água carregados pela correnteza, junto ao substrato e na superfície da água, principalmente na região central do igarapé. Durante o processo de captura de itens arrastados pela correnteza, os peixes nadavam orientados contra a correnteza; após inspecionarem o item (podendo ou não capturá-lo), deixavam-se levar pela correnteza, para novamente nadar em direção à montante. Indivíduos de pequeno porte formaram grupos de até 15 indivíduos, espacialmente restritos às áreas marginais (mais rasas). Somente foram observados comportamentos alimentares individuais com ausência de comportamento agonístico e/ou forrageio conjunto.

\section{Discussão}

Igarapés de floresta de terra firme são componentes importantes do sistema fluvial amazônico (WELCOMME, 1985), que abrigam um grande número de espécies de peixes (BÜHRNHEIM; COX-FERNANDES, 2003; MONTAG etal., 2009; OLIVEIRA et al., 2009; BARROS et al., 2011) principalmente de porte pequeno, com comprimento total menor que $100 \mathrm{~mm}$ (BÜHRNHEIM, 2002). Este ambiente apresenta diversidade de habitats menor do que de rios, possuindo geralmente dois ambientes típicos: ambientes de alta velocidade de correnteza, com substrato composto principalmente por areia, seixos, raízes de plantas e troncos submersos; e ambientes de poções mais profundos, formados em áreas de baixa velocidade de correnteza, normalmente nos retornos dos meandros do canal ou após barreiras naturais (e.g. troncos de árvores), com o substrato constituído por bancos de folhas e pequenos galhos (FITTKAU, 1967; BÜHRNHEIM, 2002).

Indivíduos jovens de Knodus moenkhausii formam pequenos grupos (até oito indivíduos), espacialmente restritos às áreas mais rasas, próximas às margens, investindo em porções mais profundas do riacho na ausência de adultos (CENEVIVA-BASTOS; CASATTI, 2007). Desta forma, tal comportamento descrito para uma espécie do mesmo gênero pode ter favorecido a captura por nossos aparatos de pesca, sendo coletado um maior número de juvenis de pequeno porte $(<20 \mathrm{~mm})$, na região marginal, no período da seca e um elevado número de jovens de maior porte (de 26 a $30 \mathrm{~mm}$ ) no período chuvoso, estação na qual os igarapés apresentam maior 
profundidade por influência de chuvas locais (NAIMAN; BILBY, 1998; CARVALHO, 2008).

Igarapés possuem águas pobres em nutrientes e o denso dossel da floresta impede a penetração da luz na superfície dos mesmos, sendo a presença de plantas aquáticas praticamente inexistente (JUNK; FURCH, 1985; WALKER, 1995). Nestes ambientes oligotróficos, onde a produtividade primária é baixa, a principal fonte energética para sustentar as comunidades é baseada na entrada de folhas, flores, artrópodes terrestres e larvas de insetos (GOULDING, 1980; WELCOMME, 1985; GOULDING et al., 1988; WALKER, 1991; CASTRO, 1999; MOULTON; MAGALHÃES, 2003; ABILHOA et al., 2009) no ambiente aquático. Estudos sobre dieta de peixes em igarapés amazônicos apontam que macroinvertebrados constituem o grupo de presas mais importante nas teias tróficas desses sistemas aquáticos (KNÖPPEL, 1970; SAUL, 1975; SOARES, 1979; HENDERSON; WALKER, 1986; SILVA, 1993).

Segundo Soares (1979), peixes de riacho tropicais podem apresentar preferências por tipos específicos de itens alimentares, contudo o alimento de origem alóctone, principalmente insetos terrestres, é descrito como o mais importante. Tais resultados têm servido de suporte para generalizações sobre a importância das relações entre os peixes e a floresta ripária, que caracteriza extrema dependência de fontes alóctones de alimento para a ictiofauna de igarapés (SILVA, 1993; SABINO; ZUANON, 1998; LOWE-MCCONNELL, 1999). Peixes que apresentam pequeno porte são especialmente capacitados para explorar alimentos como artrópodes aquáticos e terrestres (MOYLE; CECH, 1996; CASTRO; MENEZES, 1998; CASTRO, 1999).

Assim como os resultados encontrados para $K$. heteresthes, Ortaz (2001) descreve que as espécies Knodus deuterodonoides e Knodus sp. apresentaram hábitos predominantemente insetívoros, consumindo maior diversidade de itens (em sua maioria alóctone) na estação chuvosa. Segundo Mazzoni et al. (2010), para estudos em riachos do alto rio Tocantins (GO), a espécie Knodus sp. foi considerada carnívora/insetívora, sendo sua dieta principalmente baseada em insetos alóctones e autóctones. Os autores supracitados caracterizaram a espécie com corpo alto, comprimido lateralmente e olhos grandes, com hábito alimentar orientado pela capacidade visual. Essa espécie tem sua dieta baseada em itens de origem animal e apresentam comportamento tanto pelágico quanto epibentônico, se alimentando na coluna d'água e/ou no substrato (MAZZONI et al., 2010), sendo estas características importante para o consumo de itens alóctones (MAZZONI; IGLESIAS-RIOS, 2002).

Nossos resultados apontaram que itens animais oriundos da floresta ripária foram predominantes na dieta de $K$. heteresthes, tanto para indivíduos jovens quanto para adultos. Wootton (1998) descreve que o crescimento dos peixes é acompanhado por importantes mudanças na dieta e na susceptibilidade à predação, sendo que o primeiro ano é um momento de intenso incremento no tamanho do corpo. O incremento ontogenético do comprimento do intestino em relação ao comprimento corporal tem sido amplamente registrado em peixes tropicais (MAZZONI et al., 2010). Esse processo está relacionado à capacidade de absorção do intestino que, por sua vez, torna-se mais elevada com o aumento da extensão do mesmo e, entre as espécies onívoras, é fortemente esperada a redução da participação dos itens alimentares de origem animal na medida em que se tornam adultos (MAZZONI et al., 2010). Apesar de não termos realizado análises relacionadas ao comprimento do intestino, apontamos que esse processo não foi registrado para o presente estudo, pois os indivíduos adultos, assim como os juvenis, apresentaram elevado consumo de itens de origem animal.

Muitas espécies de peixes utilizam os recursos de origem alóctone (frutos, sementes e insetos terrestres) como fonte principal de energia (CLARO-JR et al., 2004). Tal prática visa a captura de itens alimentares que sejam importantes para a obtenção mais eficiente dos recursos energéticos necessários à sobrevivência (ZAVALA-CAMIM, 1996). Dessa forma, supomos que a elevada ingestão de insetos por parte dos indivíduos jovens de $K$. heteresthes esteja relacionada ao desenvolvimento de tecido somático (crescimento), e nos adultos associado a alocação de energia para a produção de tecidos germinativos, considerando que foram encontrados muitos indivíduos ( $\mathrm{CP}>30 \mathrm{~mm})$ em estádio de reprodução avançado em cinco dos seis bimestres analisados (observação pessoal). 
Estudos sobre forrageamento de peixes de riachos neotropicais apontam, em sua maioria, uma dieta euritrófica, que pode ser alterada frente a mudanças no ambiente decorrentes de ações antrópicas ou mesmo ao longo de flutuações sazonais (SABINO; CASTRO, 1990). A elevada ocorrência de itens alóctones registrados durante a estação seca suporta a hipótese de vários autores (ex. WINEMILLER, 1989; LUIZ et al., 1998), onde a entrada de matéria alóctone no meio aquático é importante para o suprimento alimentar em riachos neste período. Contrariamente, diversos estudos apontam que proporções mais elevadas de itens alóctones são fornecidos aos riachos durante a estação chuvosa (MORELLATO, 1992; UIEDA; KIKUCHI, 1995; AFONSO et al., 2000; REZENDE; MAZZONI, 2005). Padrões sazonais determinados pelo regime de chuvas e ventos influenciam o transporte de itens durante eventos de tempestade (ANGERMEIER; KARR, 1983), sendo esta influência aumentada com a remoção da mata ciliar (DE LA ROSA, 1995).

Segundo Rezende e Mazzoni (2003), muitas espécies de Characidae são oportunistas quanto ao uso de recursos alimentares em ambientes de riachos, e isso mostra que certas espécies muito provavelmente utilizam um determinado tipo de item alimentar na ausência de outro de maior preferência. Ceneviva-Bastos e Casatti (2007) observaram grupos de 5-20 indivíduos de K. moenkhausii forrageando continuamente, praticando a cata de itens na coluna d'água carregados pela correnteza, a cata de itens junto ao substrato ou à vegetação submersa e a cata de itens na superfície da água, sendo tal comportamento também observado para $K$. heteresthes. A participação praticamente equitativa de itens autóctones e alóctones na dieta de K. moenkhausii é um indicativo de que esta necessita de poucas exigências quanto à integridade da vegetação marginal para sua alimentação (CENEVIVA-BASTOS; CASATTI, 2007). Tal afirmação, não corrobora com os resultados encontrados para a dieta de $K$. heterethes, que utilizou uma elevada quantidade de itens alóctones em ambas as estações, apontando que esta espécie talvez seja mais dependente da mata ripária como fonte de recurso alimentar (insetos terrestres).
A vegetação ripária é importante para a manutenção da integridade biológica de ambientes aquáticos lóticos, notavelmente por seus efeitos sobre a produtividade primária, temperatura e fornecimento de material alóctone (galhos, frutos, sementes, folhas e troncos) para dentro do canal (CASATTI et al., 2009). Estes últimos servem como alimento, sítio reprodutivo e refúgio para diversas espécies (SCHNEIDER; WINEMILLER, 2008). A supressão das matas ripárias, por sua vez, altera as relações tróficas entre os organismos (PUSEY; ARTHINGTON, 2003) e afeta a estrutura e composição das comunidades aquáticas (CASATTI et al., 2009).

Apesar de a espécie estudada consumir uma elevada diversidade de itens alimentares, não a consideramos como oportunista, quanto à utilização de recursos alimentares, pois a mesma apresentou elevada preferência por insetos terrestres, apontando de tal forma certo nível de especialização trófica. Tal especialização pode estar relacionada às elevadas abundâncias encontradas pela espécie, que possivelmente demonstra boa capacidade em alocar energia para crescimento, no caso dos indivíduos jovens, e reprodução (adultos), tornando-a uma das espécies dominantes em igarapés conservados.

\section{Agradecimentos}

Agradecemos a Marina Fonseca pela ajuda na identificação do conteúdo estomacal e a professora Dra. Carolina Rodrigues da Costa Doria por autorizar o uso do Laboratório de ictiologia e Pesca - LIP da Universidade Federal do Rondônia.

\section{Referências}

ABELL, R.; THIEME, M. L.; REVENGA, C.; BRYER, M.; KOTTELAT, M.; BOGUTSKAYA, N.; COAD, B.; MANDRAK, N.; BALDERAS, S. C.; BUSSING, W.; STIASSNY, M. L.; SKELTON, P.; ALLEN, G. R.; UNMACK, P.; NASEKA, A.; NG, R.; SINDORF, N.; ROBERTSON, J.; ARMIJO, E.; HIGGINS, J. V.; HEIBEL, T. J.; WIKRAMANAYAKE, E.; OLSON, D.; LÓPEZ, H. L.; REIS, R. E.; LUNDBERG, J. G.; PÉREZ, M. H. S.; PETRY, P. Freshwater ecoregions of the world: a new map of biogeographic units for freshwater biodiversity conservation. BioScience, Uberlândia, v. 58, n. 5, p. 406-414, 2008.

ABILHOA, V., BORMAATOWSKI, H.; OTTO, G. Temporal and ontogeneticvariations on feeding habits of Hollandichthys 
multifasciatus (Teleostei: Characidae) in coastal Atlantic rainforest streams, southern Brazil. Neotropical Ichthyology, Porto Alegre, v. 7, n. 3, p. 415-420, 2009.

AFONSO, A. A. O.; HENRY, R.; RODELlA, R. C. S. M. Allochthonous matter input in two different stretches of a headstream (Itatinga, São Paulo, Brazil). Brazilian Archives of Biology and Technology, Curitiba, v. 43, n. 3, p. 335-343, 2000.

ANGERMEIER, P. L.; KARR, J. R. Fish communities along environmental gradients in a system of tropical streams. Environmental Biology of Fishes, New York, v. 9, n. 2, p. 117-135, 1983.

BARROS, D. F.; ZUANON, J.; MENDONÇA, F. P.; ESPÍRITOSANTO, H. M. V.; GALUCH, A. V.; ALBERNAZ, A. L. M. The fish fauna of streams in the Madeira-Purus interfluvial region, Brazilian Amazon. Check List, Rio Claro, v. 7, n. 6, p. 768-773, 2011.

BRITSKI, H. A.; SILIMON, K. Z. S.; LOPES, B. S. Peixes do Pantanal: manual de identificação. Brasília: Empresa Brasileira de Pesquisa Agropecuária, 1999. 184 p.

BUCKUP, P. A. Sistemática e biogeografia de peixes de riachos. In: CARAMASCHI, E. P.; MAZZONI, R.; PERES NETO, P. R. (Ed.). Ecologia de peixes de riachos. Oecologia Brasiliensis. 4 ed. Rio de Janeiro: PPGE-UFRJ, 1999. p. 91-138.

BUCKUP, P. A.; MENEZES, N. A.; GHAZZI, M. S. Catálogo das espécies de peixes de água doce do Brasil. Rio de Janeiro: Museu Nacional, 2007. 195 p.

BÜHRNHEIM, C. M. Heterogeneidade de habitat: rasos e fundos em assembléias de peixes de igarapés de terra firme na Amazônia Central. Revista Brasileira de Zoologia, Curitiba, v. 19, n. 3, p. 889-905, 2002.

BÜHRNHEIM, C. M.; COX-FERNANDES, C. C. Structure of fish assemblages in Amazonian rain-forest streams: effects of habitats and locality. Copeia, Lawrence, v. 2003, n. 2, p. 255-262, 2003.

CARVALHO, L. N. História natural de peixes de igarapés amazônicos: utilizando a abordagem do conceito do rio continuo. 2008. 142 f. Tese (Doutorado em Biologia) - Instituto Nacional de Pesquisas da Amazônia, Manaus. 2008.

CASATTI, L.; FERREIRA, C. P.; CARVALHO, F. R. Grassdominated stream sites exhibit low fish species diversity and dominance by guppies: an assessment of two tropical pasture river basins. Hydrobiologia, Bruxelas, v. 632, p. 273-283, 2009.

CASATTI, L.; PÉREZ-MAYORGA, M.A.; CARVALHO, F.R.; BREJÃO, G.L; COSTA, I.B. 2013. The stream fish fauna from the rio Machado basin, Rondônia State, Brazil. Check List, Rio Claro, v. 9, n. 6, p. 1496-1504, 2013.

CASTRO, R. M. C. Evolução da ictiofauna de riachos sulamericanos: padrões gerais e possíveis processos causais. In: CARAMASCHI, E. P.; MAZZONI, R.; BIZERRIL, C. R. S. F.; PERES NETO, P. R. (Ed.). Ecologia de peixes de riachos: estado atual e perspectivas. 1 ed. Rio de Janeiro: PPGE-UFRJ, 1999. p. 139-155.

CASTRO, R. M. C.; CASATTI, L.; SANTOS, H. F.; FERREIRA, K. M.; RIBEIRO, A. C.; BENINE, R. C.; DARDIS, G. Z. P.; MELO, A. L. A.; STOPIGLIA, R.; ABREU, T. X.; BOCKMANN, F. A.; CARVALHO, M.; GIBRAN, F. Z.; LIMA, F. C. T. Estrutura e composição da ictiofauna de riachos do rio Paranapanema, sudeste e sul do Brasil. Biota Neutropica, Campinas, v. 3, n. 1, p. 1-14, 2003.
CASTRO, R. M. C.; CASATTI, L.; SANTOS, H. F.; MELO, A. L. A.; MARTINS, L. S. F.; FERREIRA, K. M.; GIBRAN, F. Z.; BENINE, R. C.; CARVALHO, M.; RIBEIRO, A. C.; ABREU, T. X.; BOCKMANN, F. A.; PELIÇÃO, G. Z.; STOPLIGLIA, R.; LANGEANI, F. Estrutura e composição da ictiofauna de riachos da bacia do rio Grande no estado de São Paulo, sudeste do Brasil. Biota Neutropica, Campinas, v. 4, n. 1, p. 1-39, 2004.

CASTRO, R. M. C.; CASATTI, L.; SANTOS, H. F.; VARI, R. P.; MELO, A. L. A.; MARTINS, L. S. F.; ABREU, T. X.; BENINE, R. C.; GIBRAN, F. Z.; RIBEIRO, A. C.; BOCKMANN, F. A.; CARVALHO, M.; PELIÇÃO, G. Z.; FERREIRA, K. M.; STOPIGLIA, R.; AKAMA, A. Structure and composition of the stream ichthyofauna of four tributary rivers of the upper Rio Paraná basin, Brazil. Ichthyological Exploration of Freshwaters, Cornol, v. 16, n. 3, p. 193-214, 2005.

CASTRO, R. M. C.; MENEZES, N. A. Estudo diagnóstico da diversidade de peixes do estado de São Paulo. In: CASTRO, R. M. C. (Ed.). Biodiversidade do estado de São Paulo, Brasil: síntese do conhecimento ao final do século XX. 6 ed. São Paulo: WinnerGraph, 1998. p. 1-13.

CENEVIVA-BASTOS, M.; CASATTI, L. Oportunismo alimentar de Knodus moenkhausii Teleostei, Characidae): uma espécie abundante em riachos do noroeste do estado de São Paulo. Iheringia, Série Zoologia, Porto Alegre, v. 97, v. 1, p. 7-15, 2007.

CLARO-JR, L.; FERREIRA, E.; ZUANON, J.; ARAUJO-LIMA, C. O efeito da floresta alagada na alimentação de três espécies de peixes onívoros em lagos de várzea da Amazônia Central, Brasil. Acta Amazonica, Manaus, v. 34, n. 1, p. 133-137, 2004.

DE LA ROSA, C. Middle American streams and rivers. In: CUSHING, C. E.; CUMMINS, K. W.; MINSHALL, G. W. (Ed.). Ecosystems of the World - River and stream ecosystems. 2 ed. Berkeley: University of Califórnia Press, 2006. p. 189-218.

FERNANDES, L. C.; GUIMARÃES, S. C. P. Atlas geoambiental de Rondônia. Porto Velho: Secretaria de estado do Desenvolvimento Ambiental, 2002. 151 p.

FITTKAU, E. J. On the ecology of Amazonian rain-forest streams. Atas do Simpósio sobre a Biota Amazônica, Manaus, v. 3, p. $97-$ 108, 1967.

GOULDING, M. The fishes and the forest: explorations in Amazonian natural history. Berkeley: University of Califórnia, 1980. 280 p.

GOUlding, M.; CARVALHO, L. M.; FERREIRA G. E. Rio Negro, rich life in poor water. The Hague: SPB Academic Publishing, 1998. $200 \mathrm{p}$.

HAHN, N. S.; ADRIAN, I. F.; FUGI, R.; ALMEIDA, V. L. L. Ecologia trófica. In: VAZZOLER, A. E. A. M.; AGOSTINHO, A. A.; HAHN, N. S. (Ed.). A planície de inundação do alto rio Paraná: aspectos físicos, biológicos e socioeconômicos. 1 ed. Maringá. EDUEM, 1997. p. 209-228.

HELLAWELL, J. M.; ABEL, R. A rapid volumetric method for the analysis of the food of fishes. Journal of Fish Biology, Malden, v. 3, n. 1, p. 29-37, 1971.

HENDERSON, P. A.; WALKER, I. On the leaf litter community of the Amazonian blackwater stream Tarumazinho. Journal of Tropical Ecology, Winchelsea, v. 2, n. 1, p. 1-16, 1986.

HYNES, H. B. N. The food of fresh-water sticklebacks (Gasterosteus aculeatus and Pygosteus pungitius), with a review of 
methods used in studies of the food of fishes. Journal of Animal Ecology, London, v. 19, n. 1, p. 36-57, 1950.

HYSLOP, E. J. Stomach contents analysis: a review of methods and their application. Journal of Fish Biology, Malden, v. 17, n. 4, p. 411-429, 1980.

JUNK, W. J.; FURCH, K. Key environments Amazonia. In: PRANCE, G. T.; LEVEJOY, T. E. (Ed.). The physical and chemical properties of the Amazonian waters and their relationships with the biota. 1 ed. Oxford: Pergamon Press, 1985. p. 3-17.

KAWAKAMI, E.; VAZZOLER, G. Método gráfico e estimativa de índice alimentar aplicado no estudo de alimentação de peixes. Boletim do Insituto Oceanógrafo, São Paulo, v. 29, n. 2, p. 205207, 1980.

KNÖPPEL, H.A. Food of central Amazon fishes. Contribution to the nutrient-ecology of amazonian rain-forest streams. Amazoniana, Plön, v. 2, n. 3, p. 257-352, 1970.

KRUSCHE, A. V.; BALLESTER, M. V. R.; VICTORIA, R. L.; BERNARDES, M. C.; LEITE, N. K.; HANADA, L.; VICTORIA, D. C.; TOLEDO, A. M.; OMETTO, J. P.; MOREIRA, M. Z.; GOMES, B. M.; BOLSON, M. A.; NETO, S. G.; BONELLI, N.; DEEGAN, L.; NEILL, C.; THOMAS, S.; AUFDENKAMPE, A. K.; RICHEY, J. E. Efeitos das mudanças do uso da terra na biogeoquímica dos corpos d'água da bacia do rio Ji-Paraná, Rondônia. Acta Amazonica, Manaus, v. 35, n. 2, p. 197-205, 2005. LEHNER, C. F. The beauty of small flies. Nature Cell Biology, London, v. 1, n. 5, p. E129-E130, 1999.

LÉVÊQUE, C.; OBERDORFF, T.; PAUGY, D.; STIASSNY, M. L. J.; TEDESCO, P. A. Global diversity of fish (Pisces) in freshwater. In: BALIAN, E. V.; LÉVÊQUE, C.; SEGERS, H.; MARTENS, K. (Ed.). Freshwater animal diversity assessment. 1 ed. New York: Springer, 2008. p. 545-567.

LOWE-MCCONNELL, R. H. Ecological studies in tropical fish communities. New York: Press Syndicate of de University of Cambridge. 1987. 382 p.

LOWE-MCCONNELL, R. H. Estudos ecológicos de comunidades de peixes tropicais. São Paulo: EDUSP, 1999. 584 p.

LUIZ, E. A.; AGOSTINHO, A. A.; GOMES, L. C.; HAHN, N. S. Ecologia trófica de peixes em dois riachos da bacia do rio Paraná. Revista Brasileira de Biologia, São Carlos, n. 58, v. 2, p. 273-285, 1998.

MAZZONI, R.; ARAÚJO, R. R. S.; SANTOS, G. C. T.; IGLESIAS-RIOS, R. Feeding ecology of Phalloceros anisophallos (Osteichthyes: Cyprinodontiformes) from Andorinha Stream, Ilha Grande, Brazil. Neotropical Ichthyology, Porto Alegre, v. 8, n. 1, p. 179-182, 2010.

MAZZONI, R.; IGLESIAS-RIOS, R. Environmentally related life history variations in Geophagus brasiliensis. Journal of Fish Biology, Malden, v. 61, n. 6, p. 1606-1618, 2002.

MONTAG, L. F. A.; ALBUQUERQUE, A. A.; FREITAS, T. M. S.; BARTHEM, R. B. Ictiofauna de campos alagados da Ilha do Marajó, estado do Pará, Brasil. Biota Neotropica, Campinas, n. 9, v. 3, p. 241-253, 2009.

MORELLATO, L. P. C. Sazonalidade e dinâmica de ecossistemas florestais na Serra do Japi. In: MORELLATO, L. C. P. (Ed.). História natural da Serra do Japi: ecologia e preservação de uma área florestal no Sudeste do Brasil. 1. ed. Campinas: UNICAMP, 1992. p. $98-110$.
MOUlTON, T. P.; MAGAlHÃES, S. A. P. Responses of leaf processing to impacts in streams in Atlantic Rain Forest, Rio de Janeiro, Brazil - A test of the biodiversity ecosystem function relationship? Brazilian Journal of Biology, São Carlos, v. 63, n. 1, p. 87-95, 2003.

MOYLE, P. B.; CECH JR, J. J. Fishes: an introduction to ichthyology. New Jersey: Prentice Hall, 1996. 726 p.

NAIMAN, R. J.; BILBY, R. E. River ecology and management: lessons from the Pacific Coastal Ecoregion. New York: Springer, 1998. $705 \mathrm{p}$

NUÑEZ, J.; DUPONCHELLE, F. Towards a universal scale to assess sexual maturationand related life history traits in oviparous teleost fishes. Fish Physiology and Biochemistry, Amsterdam, v. 35, n. 1, p. 167-180, 2008.

OLIVEIRA, R. R.; ROCHA, M. S.; ANJOS, M. B.; ZUANON, J.; PY-DANIEL, L. H. R. Fish fauna of small streams of the CatuaIpixuna Extractive Reserve, State of Amazonas, Brazil. Check List, Rio Claro, v. 5, n. 2, p. 154-172, 2009.

ORTAZ, M. Diet seasonality and food overlap among fishesof the upper Orituco stream, northern Venezuela. Revista de Biología Tropical, San José, v. 49, n. 1, p.191-197, 2001.

PUSEY, B. J.; ARTHINGTON, A. H. Importance of the riparian zone to the conservation and management of freshwater fish: a review. Australian Journal of Marine and Freshwater Research, Melbourne, v. 54, n.1, p. 1-16, 2003.

REIS, R. E.; KULLANDER, S. O.; FERRARI JR, C. J. Check list of the freshwater fishes of South and Central America. Porto Alegre: EDPUCRS, 2003. 742 p.

REZENDE, C. F.; MAZZONNI, R. Aspectos da alimentação de Bryconamericus microcephalus (Characiformes, Tetragonopterinae) no Córrego Andorinha. Biota Neotroprica, Campinas, v. 3, n. 1, p. $1-6,2003$.

REZENDE, C. F.; MAZZONI, R. Seasonal variation in the input of allochthonous matter in an Atlantic Rain Forest stream. Acta Limnologica Brasiliensia, Rio Claro, v. 17, n. 2, p. 167-175, 2005. SABINO, J.; CASTRO, R. M. C. Alimentação, período de atividade e distribuição espacial dos peixes de um riacho da floresta Atlântica (Sudeste do Brasil). Revista Brasileira de Biologia, São Carlos, v. 50, n. 1, p. 23-36, 1990.

SABINO, J.; ZUANON, J. A. A stream fish assemblage in Central Amazonia: distribution, activity patterns and feeding behavior. Ichthyological Exploration of Freshwaters, Cornol, v. 8, n. 3, p. 201-210, 1998.

SAUL, W. G. An ecological study of fishes at a site in upper Amazonian Ecuador. Proceedings of the National Academy of Sciences of the United States of America, Washington, v. 127, p. 93-134, 1975.

SCHNEIDER, K. N.; WINEMILLER, K. O. Structural complexity of woody debris patches influences fish and macroinvertebrate species richness in a temperate floodplain-river system. Hydrobiologia, Bruxelas, v. 610, p. 235-244, 2008.

SHIBATTA, O. A.; ORSI, M. L.; BENNEMANN, S. T.; SILVASOUZA, A. T. Diversidade e distribuição de peixes na bacia do rio Tibagi. In: MEDRI, M. E.; BIANCHINI, E.; SHIBATTA, O. A.; PIMENTA, J. A. (Ed.). A bacia do rio Tibagi. 1. ed. Londrina: EDUEL, 2002. p. 403-423. 
SILVA, C. P. D. Alimentação e distribuição espacial de algumas espécies de peixes do Igarapé do Candirú, Amazonas, Brasil. Acta Amazonica, Manaus, v. 23, n. 2, p. 271-285, 1993.

SOARES, M. G. M. Aspectos ecológicos (alimentação e reprodução) dos peixes do igarapé do Porto. Acta Amazonica, Manaus, v. 9, n. 2, p. 325-325, 1979.

UIEDA, V. S.; KIKUSHI, R. M. Entrada de material alóctone (detritos vegetais e invertebrados terrestres) num pequeno curso d'água corrente na Cuesta de Botucatu, SP. Acta Limnologica Brasiliensia, Rio Claro, v. 7, n. 1, p. 105-114, 1995.

VARI, R. P.; MALABARBA, L. R. Neotropical ichthyology: an overview. In: MALABARBA, L. R.; REIS, R. E.; VARI, R. P.; LUCENA, Z. M. S.; LUCENA, C. A. S. (Ed.). Phylogeny and classification of Neotropical fishes. 1 ed. Porto Alegre: EDPUCRS, 1998. p. 1-11.

WALKER, I. Algumas considerações sobre um programa de zoneamento da Amazônia. In: VAL, A. L.; FIGLIUOLO, R.; FELDBERG, E. (Ed.). Bases científicas para estratégias de preservação e desenvolvimento da Amazônia. 1. ed. Manaus: INPA, 1991. p. 37-46.
WALKER, I. Amazonian streams and small rivers. In: TUNDISI, J. G.; BICUDO, C. E. M.; MATSUMURA-TUNDISI, T. (Ed.). Limnology in Brazil. 3. ed. Rio de Janeiro: Academia Brasileira de Ciências, Sociedade Brasileira de Limnologia, 1995. p. 167-193. WELCOMME, R. L. River fisheries. Rome: FAO Fisheries Technical Paper, 1985. 330 p.

WINEMILLER, K. O. Ontogenetic diet shifts and resource partitioning among pscivorous fishes in the Venezuelan ilanos. Environmental Biology of Fishes, New York, v. 26, n. 2, p. 177199, 1989.

WOOTTON, R. J. Ecology of teleost fishes. London: Chapman and Hall, 1998. 386 p.

ZAVALA-CAMIN, L. A. Introdução ao estudo sobre alimentação natural em peixes. Maringá: EDUEM, 1996. 129 p. 